\title{
Assessing a potential solution for spatially referencing of historical aerial photography in South Africa
}

\author{
Michele Denner, ${ }^{\mathrm{a}}$ Jacobus H. Raubenheimer ${ }^{\mathrm{b}}$ \\ ${ }^{a}$ Department of Rural Development and Land Reform, Chief Directorate: National Geospatial Information, Mowbray, Western \\ Cape, South Africa; michelle.denner@drdlr.gov.za \\ ${ }^{b}$ Cape Peninsula University of Technology,Bellville, Western Cape, South Africa; RaubenheimerJ@cput.ac.za
}

\begin{abstract}
Historical aerial photography has become a valuable commodity in any country, as it provides a precise record of historical land management over time. In a developing country, such as South Africa, that has undergone enormous political and social change over the last years, such photography is invaluable as it provides a clear indication of past injustices and serves as an aid to addressing post-apartheid issues such as land reform and land redistribution. National mapping organisations throughout the world have vast repositories of such historical aerial photography. In order to effectively use these datasets in today's digital environment requires that it be georeferenced to an accuracy that is suitable for the intended purpose. Using image-to-image georeferencing techniques, this research sought to determine the accuracies achievable for ortho-rectifying large volumes of historical aerial imagery, against the national standard for ortho-rectification in South Africa, using two different types of scanning equipment. The research conducted four tests using aerial photography from different time epochs over a period of sixty years, where the orthorectification matched each test to an already ortho-rectified mosaic of a developed area of mixed land use. The results of each test were assessed in terms of visual accuracy, spatial accuracy and conformance to the national standard for ortho-rectification in South Africa. The results showed a decrease in the overall accuracy of the image as the epoch range between the historical image and the reference image increased. Recommendations on the applications possible given the different epoch ranges and scanning equipment used are provided.
\end{abstract}

Keywords: Historical aerial photography, Image-to-image georeferencing, PCI Geomatics HAP software, orthorectification

\section{Introduction}

National mapping organisations exist throughout the world simply because the various economies of the world require basic geographic information to support economic and social growth. These organisations require systems and processes designed to deliver highly accurate geospatial information, including that which gives historical con-text to land administration over time. In many instances, such information forms the basis for sound-decision making by all those interested in the management of land resources. South Africa, in particular, was plagued by various discriminatory land administration exploits following the colonial practices and more recently the apartheid era. Thus, the availability of historical data serves as a key resource when making decisions on redress and compensation for past injustices. Furthermore, aerial photography provides a visual basis to support national government in the administration of various key legislations pertaining to the sustainable management of its strategic re-sources, such as water, mining, land and coastal.

The current repository of pre-1990 aerial photography held by South Africa's national mapping organisation remains largely unusable in a geographical information system. Most of these records reside within the organisational archive as analogue aerial photography stored in film canisters. From the early 1990's onwards, analogue aerial photography was acquired for the purposes of producing ortho-rectified imagery. Such aerial photography, largely flown at a nominal scale of 1:30 000, was scanned to a 0.75 metre spatial resolution, or 25 microns, using a photogrammetric scanner. The resultant orthophotos were generated from, amongst others, a combination of post photography ground control points, photo control from aerial triangulation and digital elevation models. These ortho-rectified images conformed to South Africa's national standard where "the positional accuracy of the pixels in the horizontal does not exceed five metres at 95\% confidence level" (Department of Rural Development \& Land Reform, 2010).

With the advent of digital aerial cameras, the scanning of analogue aerial photography, for the purposes of orthorectification, was no longer required. As a result, the photogrammetric scanner was decommissioned. The current procedure for scanning of historical analogue aerial photography is undertaken using an Epson 10000XL Expression scanner, where the film is scanned at 1200 dots per inch or an approximate spatial resolution of 0.60 metres.

\section{Methods for spatially referencing of imagery}

Aerial photography is one of the main components underpinning the photogrammetric process. The spatial in-formation generated through photogrammetry allows 
one to analyse and interpret, as is the case with an orthorectified image. Bhatta (2011) notes that the accuracy of a spatially corrected aerial photograph provides an indication of the quality of the measurements that can be taken from it. It also provides an indication of the correct-ness of the approach.

\subsection{Ortho-rectification of imagery}

Generally, an ortho-rectified image is, according to Bhatta (2011) defined as one in which all the displacements caused due to the movement of the aircraft during imaging, are removed. Displacements such as the earth's curvature, distortion of the camera lens, tilting of the camera during flying and changes in the height of the topography are addressed. An ortho-rectified image is achieved through a combination of stereoscopic pairs of images, camera calibration information, exterior orientation parameters, and location of ground control, photo control and digital elevation models. The inclusion of the digital elevation models into the process allows for each pixel in the image to be projected onto a flat plane (Bhatta, 2011). Thus, an ortho-rectified image, through being referenced to a system of co-ordinates, can easily be absorbed into a geographical information system. The removal of all the displacements allows for a good degree of accuracy in the measurement of distances, areas and the accurate positioning of features on the earth. It must, however, be borne in mind that the accuracy of the final ortho-rectified image is largely dependent on the accuracy of the input information (Bhatta, 2011).

\subsection{Geo-referencing of imagery}

Georeferencing is a process of allocating geographical co-ordinates to data such that multiple datasets may be overlaid in a GIS. Bhatta (2011) cites various techniques for georeferencing, including image-to-ground georeferencing. The first technique involves the matching of a digital image to a series of surveyed ground control, obtained using conventional surveying methods. The second technique occurs when ground control points are measured on a map and then used to georeference the image. A third technique, according to Bhatta (2011), involves the matching of a system of coordinates on one image to another image covering the same area, in a process referred to as image-to-image georeferencing. In this instance, one image serves as a reference image and is used to georeference the second image. Bhatta (2011) notes that many applications involving remotely sensed data utilize this third technique, as it presents two images of the same photographic area, from different timeframes and sensor systems, which may then be used to note changes using pixel-to-pixel comparisons.

\subsection{Rubber sheeting of imagery}

Although not a uniform process, rubber sheeting, as an effective method for correcting most of the distortions in an image, is, according to Bhatta (2011), founded on the basis of the movement of a feature to a new location, in accordance with a set of defined control points. A common example would be to rubber sheet a photographic image using a map as reference. This method is not as accurate as an ortho-rectification in that distortions relating to the camera, flight of aircraft and earth curvature, amongst others, are not removed in the process. This method simply aligns a point of an image, for instance, to its corresponding point on a map. This method is useful in that it provides for a comparison and overlaying of maps and other spatial information from different epochs.

\section{Factors affecting the ortho-rectification of historical aerial photography}

Many factors may impact on the accuracy of the orthorectification of aerial photography. These include the availability of camera calibration data, photo control and suitable digital elevation models. The improper preservation of the original film, the lack of critical camera information and poor quality and quantities of ground control points are cited as potential problems in being able to utilize historical aerial photography effectively (Aguilar, Aguilar, Fernández, I. \& Mills, 2013). Poor maintenance of the film prior to scanning can influence tonal matching be-tween adjoining strips, producing visible artefacts and image blurring, particularly in steep areas (Paine \& Kiser, 2012).

The resultant accuracy of the ortho-rectified image is largely dependent on the quality of the scanning. Research (Ayhan, Erden, Atay \& Tunç, 2006) undertaken to test the factors influencing the accuracy of an orthophoto, used five areas with different photographic scales, grey tones and topography, proving that the accuracy of an orthopho-to is directly proportional to an increase in the scanning resolution. Dajun, Gong, Guan, \& Zhang (2002) note the role played by highly accurate photogrammetric scanners in improving the accuracy of the final orthophoto. This research purports the importance of selecting an appropriate scanning resolution to accurately preserve the original image quality. Increasing the scanning resolution notably impacts on the file storage space, and thus it is necessary to ensure a balanced approach between preserving the quality of the original photography in a digital image against the impacts on storage space. Luman, Store \& Hunt (1997) investigated a pilot project for the scanning of historical aerial photography, demonstrating that the use of high quality scanners to precision scan the photography provided the optimum solution for preserving such records. Further research (Grabmaier, Tempfli, Scientific Staff of ITC, Netherlands \& Messelu, 1996) into the interpretation of detail from scanned aerial photography noted degradation in features between the film and its digital counterpart, resulting directly from variations in the scanning resolution. Scanning resolutions had to be adjusted relative to the nominal scale of the imagery and the desired image interpretation. Prior research has shown that undertaking the scanning of an archive of historical aerial photography can be both time consuming and expensive, and thus the processes involved need to be effective. It is clearly more advantageous to use photogrammetric scanning 
equipment for this purpose. Utilizing less accurate scanning equipment does appear to impact on the results of the ortho-rectification process.

Further factors impacting on the accuracy of the orthorectification of historical aerial photography includes the recovery of the interior and exterior orientation parameters, when using ground control points from recent photography for transferring onto a historical dataset. A study (Vassilaki, Ioannidis \& Stamos, 2012) using a dataset of historical aerial photography with an epoch range of 65 years, purported the use of auxiliary historical maps to identify features, particularly if the epoch of the maps is closer to the age of the aerial photography.

Problems could also be experienced when orthorectifying historical panchromatic aerial photography using its colour counterpart for the transferring of the control.

\section{Overview of the PCI Geomatics HAP workflow}

As an aid to the ortho-rectification of historical aerial photography, PCI Geomatics (2013) introduced the Histor-ical Aerial Photography (HAP) workflow, as part of its Ortho Engine suite of software. This workflow would essentially address a market need for orthorectifying large repositories of historical aerial photography. For South Africa's national mapping organisation it could provide a workable solution for converting its archives of historical aerial photography into a digital environment.

The workflow itself provides three key phases of processing whereby the scanned imagery is ingested, and, using the camera calibration information, ancillary flight details, fiducial marks, digital elevation models and a reference mosaic of an area, ground control points and tie points are automatically collected in a multipath approach to continuously refine the alignment of the historical aerial photography to acceptable levels of accuracy. With the alignment completed, the imagery can then be orthorectified and mosaiced.

\begin{tabular}{|c|c|c|}
\hline $\begin{array}{c}\text { INGEST } \\
\text { PHOTOGRAPHY }\end{array}$ & $\begin{array}{c}\text { COARSE } \\
\text { ALIGNMENT }\end{array}$ & $\begin{array}{c}\text { FINE ALIGNMENT } \\
\text { AND MOSAIC }\end{array}$ \\
\hline $\begin{array}{c}\text {-Raw scanned aerial } \\
\text { photography } \\
\text { - Metadata data file } \\
\text { txt } \\
\text { - Refence digital } \\
\text { elevation model } \\
\text { - Reference image } \\
\text { mosaic }\end{array}$ & $\begin{array}{c}\text { - Camera calibration } \\
\text { - Image co-ordinates } \\
\text { - Fiducial marks } \\
\text {-Automated GCP/TP } \\
\text { collection }\end{array}$ & $\begin{array}{c}\text {-Inspection of } \\
\text { GCP/TP } \\
\text {-refining radius for } \\
\text { searching to } \\
\text { improve GCP/TP } \\
\text { collection } \\
\text {-Generate mosaic } \\
\text { and colour } \\
\text { balancing }\end{array}$ \\
\hline
\end{tabular}

Fig. 1. The HAP workflow (Adapted from PCI Geomatics, 2016)

\section{Case study}

The main objective of this research was to assess a method of ortho-rectifying historical aerial photography using a technique of image-to-image georeferencing. The research is designed to generate primary data from a methodological study (Mouton, 2001: 144), that seeks to provide solutions to explorative and evaluative questions, by conducting four tests on aerial photography covering an area outside the Cape Town city bowl. The resultant imagery would be assessed to determine the extent to which it meets the national standard for ortho-rectified imagery in South Africa. The research focused only on the scanning and ortho-rectification of historical aerial photography at an approximate nominal scale of 1:30 000. Although smaller scales of historical aerial photography exist within the film archive, these are not readily used to ortho-rectify an image.

The timeframe for the photography used in this research was from 1953 to 2014, where the 2014 epoch of imagery was used as the georeferenced mosaic for the image-toimage matching (Bhatta, 2011). Four tests were undertaken, each generating an ortho-rectified mosaic, as follows:

- In Test 1, the panchromatic aerial photography, from 2001 epoch, scanned with a photogrammetric scanner to a 0.75 metre spatial resolution was ortho-rectified and mosaiced.

- In Test 2, the same panchromatic aerial photography from 2001 epoch, scanned with an Epson 10000XL Expression scanner, at 1200 dots per inch, or 0.60 metre spatial resolution, was ortho-rectified and mo-saiced.

- In Test 3, panchromatic aerial photography, from 1992 epoch, scanned on the Epson 10000XL Expression scanner, at the same spatial resolution as in Test 2, was ortho-rectified and mosaiced.

- In Test 4, panchromatic aerial photography, from 1953 epoch, scanned on the Epson 10000XL Expression scanner, at the same spatial resolution as Tests 2 and 3 above, was orthorectified and mosaiced.

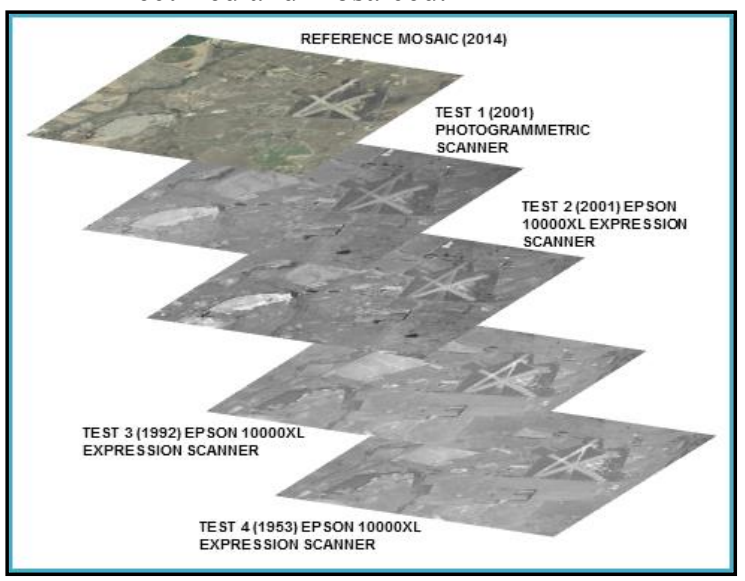

Fig. 2. A graphical representation of the four tests undertaken using different epochs and scanning equipment

\subsection{Study site}

The study site comprises of a largely developed area outside of Cape Town, approximately 25 kilometres long by 15 kilometres wide. The area has undergone significant change over the last sixty years in terms of population growth and industrial expansion. The area comprises of mixed land uses such as urban, farming, 
recreational golf courses, nature reserves, oil refineries, retail and various types of transport, including an airfield. The earliest imagery shows very little development. A visual inspection of the photography shows that most of the development has taken place post-2000. The area thus represents an opportunity to test the ability of the software for the collection of ground control points and tie points over time and in areas where development has taken place.

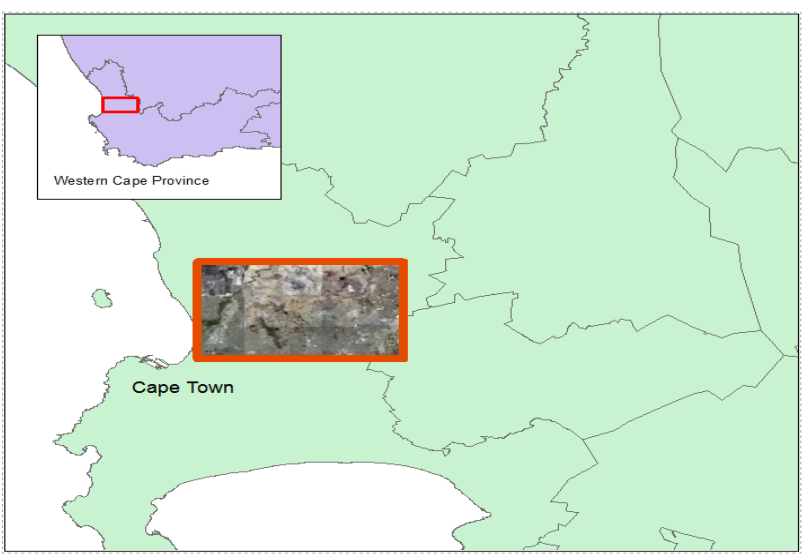

Fig. 3. A graphical representation of the study site.

\subsection{Reference datasets}

The reference datasets such as the image mosaic and digital elevation models was geo-referenced on the Hartebeesthoek94 Datum on WGS84 reference ellipsoid and projected on the Gauss Conformal projection. The software utilizes these reference datasets to complete the process of ortho-rectification.

\subsubsection{Camera calibration information}

The camera calibration information for each camera used was sourced from the original calibration certificates. The rotation of the camera panel information, relative to the rotation of the images, proved crucial in the success of the automated fiducial collection process of the HAP software. Some of the strips within the tests were rotated in-correctly and were thus excluded from the automated fiducial collection process. The process of automatic fiducial mark collection was also influenced by the quality of the scanning. It was often necessary to revert to the collection of only four fiducial marks, even though eight fiducials existed on the scanned images.

\subsubsection{Digital elevation model}

A 25 meter raster digital elevation model was obtained from the national mapping agency for the entire test area, and used in each test conducted. This model was photogrammetrically compiled from aerial photography. The accuracy thereof is such that the mean error of heights is no more than 1.7 metres at $1 \Omega$ and 3.4 metres at $2 \Omega$.

\subsubsection{Reference mosaic}

The reference mosaic was generated from aerial imagery, imaged in the red, green and blue bands, at a 0.50 meter spatial resolution, from the 2014 epoch. According to the national standard (Department of Rural Development and Land Reform, 2010) the horizontal accuracy of the pixels constituting the imagery is no greater than three metres, at a $95 \%$ confidence level.

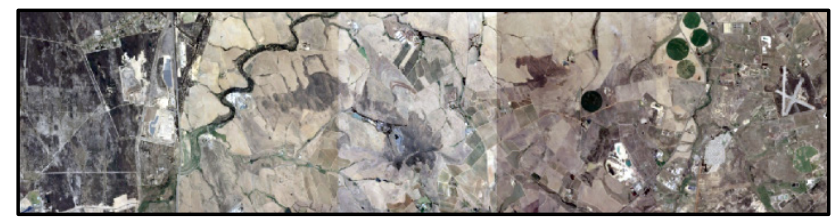

Fig. 4. A portion of the mosaiced imagery from the 2014 epoch that served as the reference image mosaic.

\subsection{Georeferencing of the historical imagery}

The process of georeferencing of each historical dataset follows on from the ingestion of the imagery and the importing of the exterior orientation parameters. Initially, a process known as nominal georeferencing enables the historical imagery to be positioned within some hundred metres of their actual position (PCI Geomatics, 2016). It further allows for problems relating to the positional accuracy, scale and orientation of the ingested historical imagery to be addressed, relative to the reference mosaic. Rotation of images according to the flight direction may al-so be resolved. Problems with the scale could result due to incorrect flying altitudes, focal length or incorrect dimensions of the aerial photography in the metadata file (PCI Geomatics, 2016). Once such errors are resolved the pro-cess of nominal georeferencing has to be redone.

Subsequent to the completion of the nominal georeferencing, two types of alignment, which when sequentially completed, apply image-to-image matching techniques to constantly improve the positional accuracy and orientation of the historical aerial images. These processes, according to PCI Geomatics (2016), collect ground control points and tie points in a multipath approach to constantly refine and align the historical aerial photography to acceptable levels of accuracy. The initial alignment, being the coarse alignment may be completed several times to improve on the residual errors. Manual editing of the ground control points and tie points can be done to improve the overall model. A second alignment process, known as fine alignment, produces the final positioning of the historical aerial images that may then be used in the ortho-rectification and mosaicking processes. A digital elevation model may also be produced prior to the ortho-rectification.

\begin{tabular}{l|r|r} 
& $\begin{array}{r}\text { Ground } \\
\text { Control } \\
\text { Points } \\
\text { collected }\end{array}$ & $\begin{array}{r}\text { Tie } \\
\text { points } \\
\text { collected }\end{array}$ \\
\hline $\begin{array}{l}\text { Test 1 2001 Photogrammetrically } \\
\text { scanned }\end{array}$ & 5286 & 3450 \\
$\begin{array}{l}\text { Test 2 2001 High resolution } \\
\text { scanner }\end{array}$ & 3441 & 2374 \\
$\begin{array}{l}\text { Test 3 1992 High resolution } \\
\text { scanner }\end{array}$ & 1958 & 3742 \\
$\begin{array}{l}\text { Test 4 1953 High resolution } \\
\text { scanner }\end{array}$ & 4 & 1951 \\
\end{tabular}


Table 1. Ground control points and tie points automatically collected for each test

\section{Results}

The main objectives of the study were to assess whether the current methods of scanning of analogue historical aerial photography were sufficient to provide a potential solution for ortho-rectifying of historical aerial photography using image-to-image processing techniques and to assess the extent to which such ortho-rectified imagery met the national standard in South Africa. The results of each test were compared to imagery that already con-formed to the national standard. Four approaches were used to assess the accuracy to ensure a visually acceptable product that conformed to a certain level of accuracy. For each test conducted, it included a visual assessment of the imagery, a comparison of the cadastre overlay, an assessment of the displacement of randomly placed points and a comparison of the number of ground control points and tie points collected for each epoch tested.

At this stage, it must be noted that the results of Test 4, where historical aerial photography from the 1953 epoch was georeferenced, indicated that only four ground control points were collected after repeated processes of coarse and fine alignments. The results of this test provided a root mean square of the ground control points of over 200 metres. The main reason for this could be the extent to which development has taken place in the area over the last 60 years, resulting in poor matching between the 1953 epoch of historical aerial imagery and the 2014 epoch reference mosaic.

As an alternative test on this epoch of imagery, the orthorectified mosaiced image from Test 1, 2001 epoch and photogrammetrically scanned, was substituted as the reference mosaic. The test was recomputed, without any improvement in the results. PCI Geomatics (2016) advises the use of a reference image that is as close as possible to the epoch of historical aerial photography to be ortho-rectified. However, generating a reference mosaic from an earlier epoch over the study site could negatively influence the accuracies achievable, given that this reference mosaic would be produced from aerial photography that was not scanned with a photogrammetric scanning device.

\subsection{Visual accuracy assessment}

Following the generation of mosaiced orthophotos for each test, a visual inspection of each mosaic was done in order to identify any anomalies resulting from the orthorectification. A few prominent features were inspected on each epoch of imagery to ascertain that each image was projected correctly and that the ortho-rectification and mosaicking was visually accurate. No noticeable problems were found to be present for the results of Test 1 or 2. However, on Test 3, slight artefacts, where portions of the panel information were found, possibly produced during the mosaicking process.

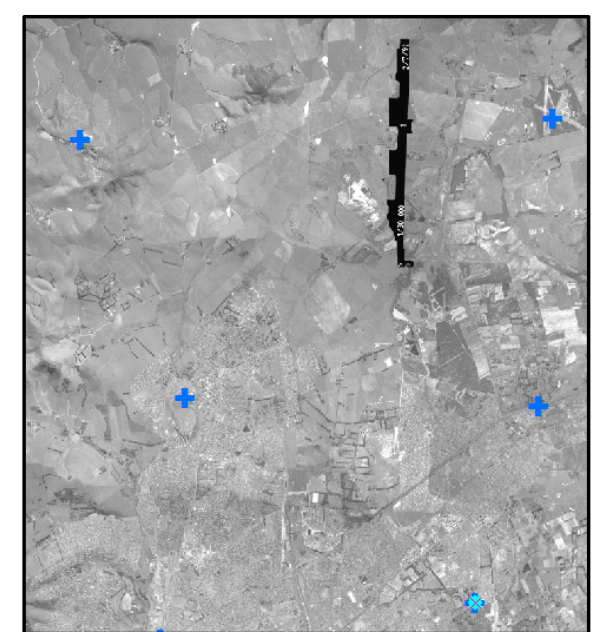

Fig. 5. A section of the mosaic for Test 3 with slight artefacts present.

\subsection{Cadastre overlay assessment}

The cadastral data overlay on each test showed a more pronounced mismatch in the earliest epoch of imagery. This was particularly noticeable along the boundaries of a local airstrip.

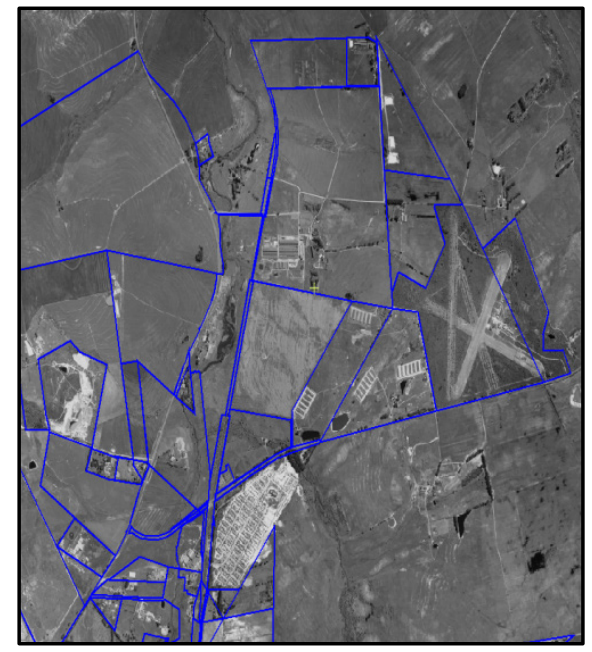

Fig. 6. A section of the mosaic generated in Test 1.

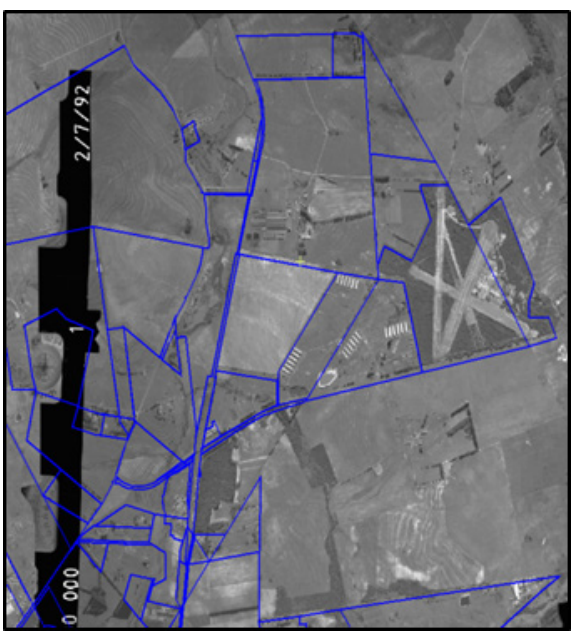

Fig. 7. A section of the mosaic generated in Test 3 showing a noticeable displacement of the image against the cadastral overlay. 


\subsection{Accuracy assessment of random points}

An accuracy assessment was done by placing nine welldefined points across the geographical area. These points had to be identifiable on each epoch of photography. The points were selected on the reference imagery and the coordinates recorded as well as the co-ordinates of the corresponding points on each test. Examples of points selected included the intersections of roads, farm fences, tanks at an oil refinery and a corner of a local airstrip. This assessment provides an indication of the displacement of each mosaic image from the reference image. Joins were computed for each point between the reference image and each test.

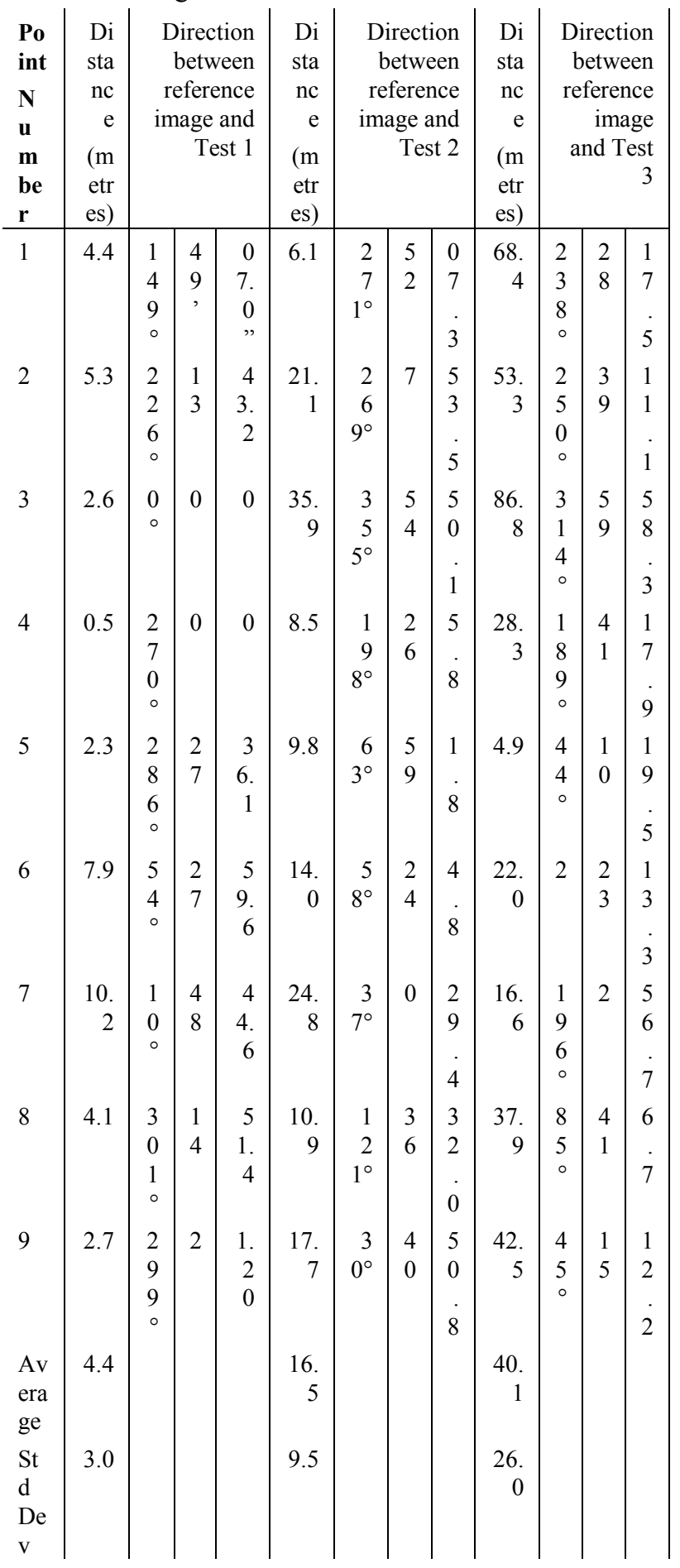

Table 2. Direction and distance offsets between reference image and each test

The average distance offset results for Test 1 , as seen in Table 2 above, indicates that the imagery scanned with a photogrammetric scanner conforms to the national standard. However, the results obtained for Test 2 and 3, where scanning was not done on a photogrammetric scanner, are inconsistent with the national standard for ortho-rectified imagery in South Africa.

\section{Conclusions}

The assessment of a potential solution for the orthorectification of historical aerial photography, such that the horizontal accuracy of the pixels in the ortho-rectified imagery should not be greater than five metres, at a $95 \%$ confidence level, has shown that acceptable results are achievable in the instance where the historical aerial photography has been scanned with a photogrammetric scanner. The required accuracies could not be achieved for historical aerial photography that was scanned with a lower resolution scanner.

Whilst the ortho-rectification of historical aerial photography will provide significant support to various strategic objectives of government departments, the achievable accuracies may give rise to a "second order ortho-rectified image", suitable for addressing problems relating to change detection and time series information.

\section{Recommendations}

It is recommended that further testing be undertaken to include additional ground control information that could potentially improve the accuracy of the ortho-rectification from photography that has been scanned with a nonphotogrammetric scanning device. It is further recommended that similar research be conducted in rural areas where there are fewer changes to the landscape, and where time series datasets containing ortho-rectified imagery would provide context for addressing issues of past injustices in land administration in South Africa.

\section{Acknowledgements}

We are grateful to Kevin Melhuish of MapAfrika and PCI Geomatics for providing the software and technical support for the completion of this dissertation.

\section{References}

Aguilar, M.A., Aguilar, F.J., Fernández, I. \& Mills, J.P. (2013). Accuracy assessment of commercial selfcalibrating bundle adjust-ment routines applied to archival aerial photography. The Photogrammetric Record, 28(141), 96-114.

Ayhan, E., Erden, Ö., Atay, G. \& Tunç, E. (2006). 'Digital orthophoto generation with aerial photos and satellite images and analyz-ing factors which affect accuracy.' XXIII FIG Congress. Munich, Germany. October 8-13, 2006. pp 1-14.

Bhatta, B. (2011). Remote Sensing and GIS. India: Oxford University Press. 
Dajun, L., Gong, J., Guan, Y \& Zhang, C. (2002). 'Accuracy analysis of digital orthophotos.' International Society for Photogramme-try and Remote Sensing: Volume XXIV, Part 2, Commission II. Xi'an, China. August 20-23, 2002. pp 241-243.

Department of Rural Development and Land Reform, Chief Directorate: National Geospatial Information. (2010). Standard for the Scanning of Film-based Aerial Photography (QLAS.SD.14_V1_2010.09.30). Cape Town.

Department of Rural Development and Land Reform, Chief Directorate: National Geospatial Information. (2010). Standard for the Ortho-rectification and Mosaicking of Imagery (QLAS.SD.15_V1_2010.12.17). Cape Town.

Grabmaier, K.A., Tempfli, R., Scientific Staff of ITC Netherlands, Messelu, G. (1996). 'Interpretability of scanned aerial photo-graphs.' InternationalSociety of Photogrammetry \& Remote Sensing, XXXI Congress, Commission IV, Working Group3.International Archive of Photogrammetry and Remote Sensing, Vol XXXI Part B4.

Luman, D.E., Stohr, C. \& Hunt, L. (1997). Digital reproduction of historical aerial photographic prints for preserving a deteriorating archive. Photogrammetric Engineering \& remote Sensing, 63(10), 1171-1179.

Mouton, J. (2001). How to succeed in your Master's and Doctoral Studies: A South African Guide and Resource Book. Pretoria: Van Schaik Publishers.

Paine, D.P. \& Kiser, J.D. (2012). Aerial photography and image interpretation. New Jersey: John Wiley \& Sons.

PCI Geomatics. (2013). PCI introduces Historical Airphoto Processing (HAP) workflow. http://www.pcigeomatics.com. Accessed 22 September 2016.

PCI Geomatics. (2016). Historical Airphoto Processing User Guide, Version 1.24. Ontario. Canada.

Vassilaki, D., Ioannidis, C. \& Stamos, A. (2012). 'Recovery of the geometry of historical aerial aerial photos associating self-calibration with ground control linear features'. Proceedings of the 1st EARSeL workshop on temporal analysis of satellite images. Mykonos, Greece. May 23-25, 2012. pp 202-207. 